\title{
Band strength factors with asymptotic expansion for Morse-Pekeris oscillators
}

Cite as: J. Chem. Phys. 69, 612 (1978); https://doi.org/10.1063/1.436625

Published Online: 11 August 2008

J. A. C. Gallas, H. P. Grieneisen, and B. P. Chakraborty

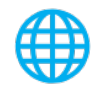

\section{ARTICLES YOU MAY BE INTERESTED IN}

The Morse oscillator in position space, momentum space, and phase space

The Journal of Chemical Physics 88, 4535 (1988); https://doi.org/10.1063/1.453761

Matrix elements for Morse oscillators

The Journal of Chemical Physics 78, 3869 (1983); https://doi.org/10.1063/1.445164

Expectation values for Morse oscillators

The Journal of Chemical Physics 79, 2537 (1983); https://doi.org/10.1063/1.446165

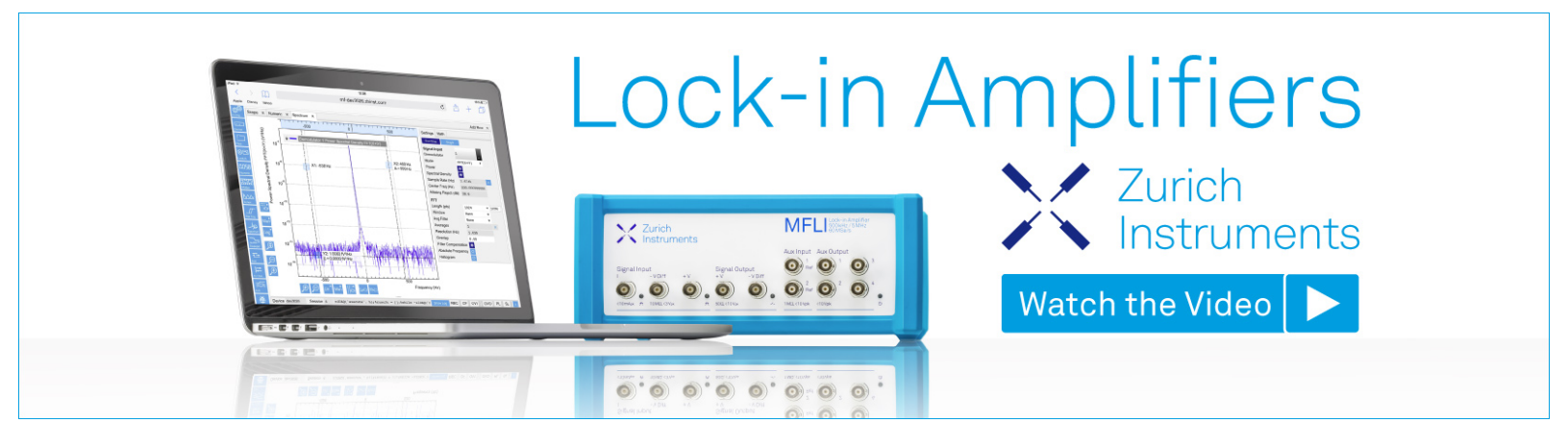




\title{
Band strength factors with asymptotic expansion for Morse-Pekeris oscillators $\left.{ }^{a}\right)$
}

\author{
J. A. C. Gallas ${ }^{\text {b) }}$ and H. P. Grieneisen \\ Instituto de Física, Universidade Federal do Rio Grande do Sul, 90.000 Porto Alegre, Brasil \\ B. P. Chakraborty
}

Instituto de Quimica, Universidade Federal do Rio Grande do Sul, 90.000 Porto Alegre, Brasil (Received 6 February 1978)

Band strength factors have been calculated by asymptotic expansion technique for $\mathrm{N}_{2}^{+}\left(B^{2} \Sigma_{u}^{+}-X^{2} \Sigma_{g}^{+}\right)$ and $N_{2}\left(C^{3} \pi_{4}-B^{3} \pi_{8}\right)$ and the rotational dependences have been studied for $J$ up to 100. A comparison between the band strengths has been made using experimentally derived electronic transition moment function for the $\mathrm{N}_{2}\left(C^{3} \pi_{u}-B^{3} \pi_{\mathrm{g}}\right)$ system. The present method of calculation does not use the usual $r$ centroid approximation.

In previous studies ${ }^{1-4}$ the asymptotic expansion method was used to calculate band strength factors when the electronic transition moment could be expressed as

$$
R_{e}(r)=A \exp (-B r) .
$$

Here we report an extension of the method which enables us to calculate band strength factors when the transition moment is expressed as

$$
R_{e}(r)=\sum a_{n} r^{n}
$$

The band strength factor is used in the derivation of transition intensities of diatomic molecules and in order to account for the thermal conditions of the source, one needs to consider the vibration-rotation interaction as well. Therefore, the present equations also include the $J$ dependence in the Morse-Pekeris model with slight modifications. ${ }^{5}$

One interesting point to note is that, due to the particular type of variable transformation involved, we do not need to use the usual $r$-centroid method $^{6}$ in the calculations.

Within the used model, the radial wavefunction for a diatomic molecule is given by

$$
\psi_{v J}(r)=N_{v J} e^{-z / 2} z^{b / 2}{ }_{1} F_{1}(-v ; b+1 ; z),
$$

where ${ }_{1} F_{1}$ is the confluent hypergeometric function. In the case considered here, of the diatomic molecule of reduced mass $\mu$, this can be written ${ }^{7}$

$$
\psi_{v J}(\eta)=N_{v J} e^{-z / 2} z^{b / 2} L_{v+b}^{b}(z),
$$

where $v$ and $J$ are the vibrational and rotational quantum numbers and

$$
\begin{aligned}
& z=K_{1} \exp \left[-a\left(r-r_{0}\right)\right], \\
& r_{0}=r_{e}(1+\alpha), \\
& \alpha=4 A B_{e} / \omega_{e}^{2}, \\
& A=B_{e} J(J+1), \\
& a=0.243559\left(\mu \omega_{e} x_{e}\right)^{1 / 2}, \\
& r_{e}=1 /\left[0.243559\left(\mu B_{e}\right)^{1 / 2}\right], \\
& K_{1}=2\left[\left(D_{2}-C_{2}\right) / \omega_{e} x_{e}\right]^{1 / 2} \\
& K_{2}=2\left(2 D_{1}-C_{1}\right) /\left(\omega_{e} x_{e} K_{1}\right), \\
& D_{1}=\omega_{e}^{2} E /\left(4 \omega_{e} x_{e}\right), \\
& D_{2}=\left(\omega_{e} E\right)^{2} /\left(4 \omega_{e} x_{e}\right), \\
& E=\exp \left(-a r_{e} \alpha\right), \\
& b=K_{2}-2 v-1, \\
& C_{1}=\left[A / a r_{0}(1+\alpha)^{2}\right]\left[4-\left(6 / a r_{0}\right)\right], \\
& C_{2}=\left[A / a r_{0}(1+\alpha)^{2}\right]\left[1-\left(3 / a r_{0}\right)\right], \\
& N_{v J}^{2}=\frac{a b}{v ! \Gamma\left(K_{2}-v\right)}, \\
& L_{v+b}^{b}(z)=L_{K_{2}-v-1}^{K} e^{-2 v-1}(z) \\
& \quad=(-1)^{v} \Gamma\left(K_{2}-v\right) \sum_{n=0}^{v}(-1)^{n}\left(\begin{array}{c}
v \\
n
\end{array}\right) \frac{z^{v-n}}{\Gamma\left(K_{2}-v-n\right)},
\end{aligned}
$$

where $\omega_{e}, \omega_{e} x_{e}$, and $B_{e}$ are the usual spectroscopic constants.

The band strength factor is defined as

$$
S_{v^{\prime} v^{\prime \prime}}^{J^{\prime \prime}}=\left|I_{v^{\prime} v^{\prime \prime \prime}}^{J^{\prime \prime}}\right|^{2}=\left|\int_{0}^{\infty} \psi_{v^{\prime} J^{\prime}}^{*} R_{e}(r) \psi_{v^{\prime \prime} J}{ }_{J} d r\right|^{2} .
$$

Substituting Eq. (1) into (2), we get

$$
\left|I_{v^{\prime} v^{\prime \prime \prime}}^{J^{\prime \prime}}\right|=\frac{N_{v^{\prime} J^{\prime}} N_{v^{\prime \prime} J^{\prime \prime}}}{a^{\prime}} \xi^{\left(K_{2}^{\prime \prime}-1\right) / 2} \sum_{\lambda=0}^{\nu^{\prime}} \sum_{\mu=0}^{v^{\prime \prime}}(-1)^{\lambda+\mu} \xi^{-\mu} B\left(b^{\prime}, v^{\prime}, \lambda\right) B\left(b^{\prime \prime}, v^{\prime \prime}, \mu\right) I_{\mu}^{\lambda}(\xi, \gamma, p),
$$

where

$$
\gamma=a^{\prime \prime} / a^{\prime}, \quad \xi=K_{1}^{\prime \prime} /\left(K_{1}^{\prime}\right)^{\gamma} \exp \left(a^{\prime \prime} r_{0}^{\prime \prime}-\gamma a^{\prime} r_{0}^{\prime}\right),
$$

a) Work supported in part by CNPq and FINEP.

b) Acknowledge gratefully a grant from CNPq.

$$
\begin{aligned}
& B(b, v, \alpha)=\frac{(b+v)_{\alpha}}{\alpha !(v-\alpha) !} \\
& (b+v)_{\alpha}=(b+v)(b+v-1) \cdots(b+v-\alpha+1), \\
& (b+v)_{0}=1,
\end{aligned}
$$

and 
TABLE I. Band strengths for the $N_{2}^{+}\left(B^{2} \Sigma_{u}^{+}-X^{2} \Sigma_{z}^{+}\right)$first negative band system.

\begin{tabular}{lccc}
\hline \hline $\begin{array}{l}\text { Band } \\
v^{\prime} v^{\prime}\end{array}$ & $J$ & From Ref. 5 & This work \\
\hline $0 \quad 0$ & 0 & 05415 & 0.5496 \\
$(3914 \AA)^{\mathrm{a}}$ & 100 & 0.5483 & 0.5553 \\
$0 \quad 1$ & 0 & 0.1978 & 0.2006 \\
$(4278 \AA)$ & 100 & 0.1924 & 0.1950 \\
$1 \quad 0$ & 0 & 0.2302 & 0.2325 \\
$(3582 \AA)$ & 100 & 0.2293 & 0.2308 \\
$0 \quad 2$ & 0 & 0.0494 & 0.0500 \\
$(4709 \AA)$ & 100 & 0.0494 & 0.0501 \\
\hline \hline
\end{tabular}

aBand heads.

$$
\begin{aligned}
& I_{\mu}^{\lambda}(\xi, \gamma, p)=\int_{0}^{\infty} z^{p} \exp \left[-\frac{1}{2}\left(z+\xi z^{\gamma}\right)\right] R_{e}(z) d z, \\
& p=\left(K_{2}^{\prime}+\gamma K_{2}^{\prime \prime}\right) / 2-(1+\gamma) / 2-1-\lambda-\gamma \mu .
\end{aligned}
$$

In the above integral we keep the symbol $R_{e}$ for the transition moment, in order to clarify its contribution, although the functional dependence is different from the previous one. This dependence can be easily found by the simple variable transformation $r$ to $z$.

Now, by transforming the variable $z=p t$, the integral can be rewritten as

$$
I_{\mu}^{\lambda}(\xi, \gamma, p)=p^{p+1} \int_{0}^{\infty} \exp [p g(t)] R_{e}(p t) d t
$$

with

$$
g(t)=-\left(t+\xi p^{\gamma-1} t^{\gamma}\right) / 2+\ln t .
$$

The integral in Eq. (4) can be evaluated by Laplace's method of asymptotic expansion, ${ }^{8}$ since $p$ is a large parameter. Then, assuming for the transition moment the form

$$
R_{e}(r)=a_{0}+a_{1} r+a_{2} r^{2}+a_{9} r^{3}+\ldots,
$$

we have, up to the second term in the expansion,

$$
\begin{aligned}
& I_{\mu}^{\lambda}(\xi, \gamma, p)=p^{p+1 / 2}\left(-\frac{\pi}{b_{2}}\right)^{1 / 2} \\
& \quad \times \exp \left(p g_{0}\right) R_{e}\left(\rho_{0}\right)\left[1+\left(E_{0}+E_{1}\right) / p\right], \\
& E_{0}=3 b_{4} / 4 b_{2}^{2}+15 b_{3}^{2} / 16 b_{2}^{3}, \\
& E_{1}=\left[3 a_{1} b_{4} / b_{2}-a_{2} / 2+\left(6 a_{2} b_{4} / b_{2}\right) \rho_{0}\right] /\left[b_{2} R_{e}\left(\rho_{0}\right)\right], \\
& g_{0}=g\left(t_{0}\right), \\
& b_{2}=g^{(2)}\left(t_{0}\right) / 2 !, \\
& b_{3}=g^{(3)}\left(t_{0}\right) / 3 !, \\
& b_{4}=g^{(4)}\left(t_{0}\right) / 4 !, \\
& \rho_{0}=r_{0}^{\prime}+\ln \left[K_{1}^{\prime} /\left(p t_{0}\right)\right] / a^{\prime},
\end{aligned}
$$

where $g^{(2)}\left(t_{0}\right), g^{(3)}\left(t_{0}\right)$, and $g^{(4)}\left(t_{0}\right)$ are the second, third, and fourth derivative of $g(t)$ evaluated for $t=t_{0}$, $t_{0}$ being the point where the first derivative of $g(t)$ is equal to zero.

Using these equations, a double precision ALGOL program has been developed for the B-6700 computer. This program has as input variables $\mu, R_{e}(r)$ and $\omega_{e}$, $\omega_{e} x_{e}, B_{e}$ for both upper and lower states, and gives as output the band strengths and, optionally, a leastsquares analysis up to the fourth degree when the $J$ dependence study is selected. The orthogonality of the wavefunctions was checked setting $R_{e}(r)=1$ and equating upper and lower spectroscopic constants: the diagonal matrix elements differ from unity by less than $10^{-6}$ and the off-diagonal elements are in the $10^{-20}$ range.

In Table I we compare the band strengths for the hypothetical $Q$ branch of the $\mathrm{N}_{2}^{+}$first negative system calculated by Shumaker ${ }^{5}$ and by the present method. The $R_{e}(r)$ expression and the needed spectroscopic constants were taken from Ref. 5. Band strengths calculated by the present method agree well with previous work as can be seen from Table $\mathrm{I}$. We also calculated band strength for the $0-0$ band of the $\mathrm{N}_{2}$ second positive system. Our values (for $J=0,0.0235$ and for $J=100$, 0.0218 ) agree quite well with values $(0.0233,0.0199)$ in Ref. 5. These last values are presented for comparison purpose only since we noted that the expression for $R_{e}(r)$ was corrected, ${ }^{9}$ a fact which Shumaker did not take into account.

Next we calculated relative band strengths for the $\mathrm{N}_{2}$ second positive system using several $R_{e}(r)$ expressions obtained experimentally but based on the $r$-centroid approximation. In Table II, which is based on Table II from Ref. 10, we summarize the used relative $R_{e}(r)$ functions. The spectroscopic constants for the $\mathrm{N}_{2} \mathrm{sec}-$ ond positive system are those given by Benesch et al. ${ }^{11}$ The calculated relative band strengths for the $Q$ branch of this band system are presented in Table III for some bands. The values are quite different from each other. However, if the calculated band strength factors using the relative $R_{e}(r)$ functions are scaled by taking the strength of the $0-0$ band equal to one, a better agreement can be obtained but not being enough. This can be attributed to the ways by which all these $R_{e}(\gamma)$ functions are obtained. Particularly, values in column (d) and (e) of Table III which differ by a factor of about 5 , when scaled, are within $10 \%$-which agree well with the fact that the $R_{e}(r)$ function of Becker, Engels, and Tatarczyk ${ }^{10}$ agrees with that of $\mathrm{Jain}^{16}$ by $\pm 5 \%$ within the definition

TABLE II. The relative $R_{e}$ functions used.

\begin{tabular}{lcc}
\hline & Relative $R_{e}(\bar{r})$ & Reference \\
\hline (a) & $-1+2.325 \bar{r}-1.102 \bar{r}^{2}$ & 12,13 \\
(b) & $1-(0.53 \pm 0.09) \bar{r}$ & 14 \\
(c) & $-1+1.9669 \bar{r}-0.8636 \bar{r}^{2}$ & 15 \\
(d) & $-1+2.1047 \bar{r}-0.9357 \bar{r}^{2}$ & 16 \\
(e) & $1-(0.51 \pm 0.03) \bar{r}$ & 10 \\
\hline
\end{tabular}


TABLE III. Relative band strengths for the $N_{2}\left(C^{3} \pi_{u}-B^{3} \pi_{f}\right)$ second positive band system. ${ }^{2}$

\begin{tabular}{|c|c|c|c|c|c|c|c|c|c|c|c|}
\hline , & $\begin{array}{l}\text { and } \\
v^{\prime \prime}\end{array}$ & $J$ & (a) & $\left(b^{0}\right)^{b}$ & (b) & $\left(b^{1}\right)^{b}$ & (c) & (d) & $\left(e^{0}\right)^{b}$ & (e) & $\left(e^{1}\right)^{b}$ \\
\hline \multirow[t]{2}{*}{0} & 0 & 0 & 0.0201 & 0.0325 & 0.0641 & 0.1065 & 0.0058 & 0.0141 & 0.0601 & .0726 & 0.0864 \\
\hline & & 100 & 0.0187 & 0.0257 & 0.0557 & 0.0971 & 0.0055 & 0.0136 & 0.0518 & 0.0639 & 0.0773 \\
\hline \multirow[t]{2}{*}{0} & 1 & 0 & 0.0147 & 0.0267 & 0.0494 & 0.0789 & 0.0041 & 0.0100 & 0.0465 & 0.0554 & 0.0649 \\
\hline & & 100 & 0.0134 & 0.0213 & 0.0423 & 0.0705 & 0.0039 & 0.0095 & 0.0397 & 0.0480 & 0.0571 \\
\hline \multirow[t]{2}{*}{1} & 0 & 0 & 0.0159 & 0.0219 & 0.0474 & 0.0828 & 0.0048 & 0.0117 & 0.0441 & 0.0544 & 0.0659 \\
\hline & & 100 & 0.0152 & 0.0173 & 0.0423 & 0.0782 & 0.0046 & 0.0114 & 0.0390 & 0.0493 & 0.0609 \\
\hline \multirow[t]{2}{*}{1} & 1 & 0 & 0.0014 & 0.0022 & 0.0045 & 0.0077 & 0.0004 & 0.0010 & 0.0042 & 0.0051 & 0.0061 \\
\hline & & 100 & 0.0014 & 0.0016 & 0.0039 & 0.0071 & 0.0004 & 0.0010 & 0.0036 & 0.0045 & 0.0056 \\
\hline \multirow[t]{2}{*}{3} & 2 & 0 & 0.0101 & 0.0126 & 0.0292 & 0.0526 & 0.0030 & 0.0074 & 0.0270 & 0.0338 & 0.0413 \\
\hline & & 100 & 0.0115 & 0.0111 & 0.0298 & 0.0574 & 0.0034 & 0.0086 & 0.0273 & 0.0352 & 0.0440 \\
\hline \multirow[t]{2}{*}{4} & 2 & 0 & 0.0104 & 0.0104 & 0.0279 & 0.0538 & 0.0032 & 0.0080 & 0.0256 & 0.0329 & 0.0413 \\
\hline & & 100 & 0.0096 & 0.0066 & 0.0221 & 0.0466 & 0.0028 & 0.0073 & 0.0199 & 0.0267 & 0.0346 \\
\hline \multirow[t]{2}{*}{5} & 4 & 0 & 0.0038 & 0.0038 & 0.0101 & 0.0192 & 0.0011 & 0.0028 & 0.0092 & 0.0118 & 0.0148 \\
\hline & & 100 & 0.0069 & 0.0047 & 0.0150 & 0.0311 & 0.0019 & 0.0049 & 0.0135 & 0.0180 & 0.0232 \\
\hline \multirow[t]{2}{*}{6} & 6 & 0 & 048 & 0.0075 & 0.0153 & 0.0258 & 0.0015 & 0.0035 & 0.0143 & 0.0174 & 0.0208 \\
\hline & & 100 & 0.0030 & 0.0057 & 0.0113 & 0.0189 & 0.0010 & 0.0024 & 0.0106 & 0.0128 & 0.0153 \\
\hline
\end{tabular}

${ }^{\mathrm{a}}$ For notation see Table II.

${ }^{b}$ The superscript 0 and 1 refers to the error limit in the relative $R_{e}(\bar{r})$ expression, i.e., for $\left(\mathrm{b}^{0}\right), R_{e}(\bar{r})=1-0.62 \bar{\gamma} ;$ for $\left(\mathrm{b}^{1}\right), R_{e}(\bar{r})=1-0.44 \bar{r} ;$ etc.

range. It is understood that the $R_{e}(r)$ functions are always derived with a convenient scaling factor. Therefore, it is felt that a more precise way of defining the transition moment is needed in order to obtain more reliable relative band strength factors.

We would also like to mention that the present method of calculation can well be used to calculate FranckCondon factors, $r$ centroids, and related integrals along with band strength factors by simply changing the constants of Eq. (5).

We have calculated relative band strength factors for the $\mathrm{N}_{2}^{+}$first negative and the $\mathrm{N}_{2}$ second positive band system for $v^{\prime}, v^{\prime \prime} \leq 8$ and for $J$ up to 100 with an increment of 10. For the majority of these bands the rotational dependence could only be well reproduced through a second degree least-squares fit in the variable $J(J$ +1 ). In view of the great number of data generated they are not published here, but are available from the authors on request.

${ }^{1}$ T. Y. Chang and M. Karplus, J. Chem. Phys. 52, 783 (1970). ${ }^{2}$ B. P. Chakraborty, Y. K. Pan, and T. Y. Chang, J. Chem.
Phys. 55, 5147 (1971).

${ }^{3}$ B. P. Chakraborty and Y. K. Pan, J. Chem. Phys. 56, 3622 (1972).

'B. P. Chakraborty and Y. K. Pan, Appl. Spectrosc. Rev. 7, 283 (1973).

${ }^{5}$ J. B. Shumaker Jr., J. Quant, Spectrosc. Radiat. Transfer 9, 153 (1969).

${ }^{6}$ P. A. Fraser, Can. J. Phys. 32, 515 (1954).

${ }^{7} \mathrm{~J}$. Rundgren, Ark. Fys. 30, 61 (1965).

${ }^{8}$ F. W. J. Olver, Asymptotic and Special Functions, (Academic, New York, 1974), Chap. III.

${ }^{9}$ L. V. Wallace and R. W. Nicholls, J. Atmos, Terrest. Phys. 24, 749 (1962).

${ }^{10}$ K. H. Becker, H. Engles, and T. Tatarczyk, Chem. Phys. Lett. 51, 111 (1977).

${ }^{11}$ W. Benesch, J. T. Vanderslice, S. G. Tilford, and P. G. Wilkinson, Astrophys. J. 142, 1227 (1965).

${ }^{12}$ L. V. Wallace and R. W. Nicholls, J. Atmos. Terrest. Phys. Phys. 7, 101 (1955).

${ }^{13}$ R. W. Nicholls, J. Atmos. Terrest. Phys. 25, 218 (1963).

${ }^{14}$ D. C. Tyte, Proc. Phys. Soc. London 80, 1347 (1962).

${ }^{15}$ D. C. Jain and R. C. Sahni, J. Quant. Spectrosc. Radiat. Transfer 7, 475 (1967).

${ }^{16}$ D. C. Jain, J. Quant. Spectrosc. Radiat. Transfer 12, 759 (1972). 